\title{
\%
}

\section{COMPETENCIAS COGNITIVAS Y DESAJUSTES EN EL MERCADO LABORAL: UNA REVISIÓN DE LA LITERATURA}

Este trabajo revisa la literatura sobre los desajustes en el mercado laboral, prestando especial atención al nivel de competencias alcanzado por los individuos como parte de su capital humano. En primer lugar, se analizan los efectos económicos del capital humano, aproximado tanto por la educación recibida como por las competencias cognitivas realmente adquiridas por los trabajadores. A continuación, nos centramos en el estudio del desajuste educativo y del desajuste en competencias, presentando distintos marcos teóricos propuestos para explicar estos desajustes, discutiendo las distintas formas de medición y revisando los resultados encontrados en la literatura empírica. Por último, se revisa la literatura más reciente que considera que individuos con un mismo nivel educativo pueden presentar competencias heterogéneas y distingue entre sobreeducación real y aparente, en función del nivel de competencias alcanzado por los trabajadores.

Palabras clave: educación, competencias cognitivas, desajuste educativo, desajuste en competencias. Clasificación JEL: I21; J23.

\section{Introducción}

En las últimas décadas, los poderes públicos han realizado un importante esfuerzo en desarrollar políticas educativas orientadas a incrementar el capital humano de la población dada la relevancia de este factor a nivel individual y sus consecuencias sobre la productividad y el

\footnotetext{
*Universidad de Extremadura.

Las autoras agradecen la financiación recibida del Ministerio de Economía y Competitividad español (Proyecto ECO2014-53702-P). Lucía Mateos Romero agradece también el apoyo del Ministerio de Educación español en el marco del programa de becas FPU.

Versión de abril de 2017.
}

crecimiento de las economías. Sin embargo, la eficiencia de estas políticas públicas puede quedar en entredicho si la economía no es capaz de obtener un rendimiento de esta inversión educativa y los recursos destinados a incrementar el capital humano de la población no se traducen en mejoras de productividad.

Este problema surge en muchas economías avanzadas como consecuencia del desempleo, y cuando los titulados universitarios ocupan puestos de trabajo de baja cualificación donde las competencias adquiridas durante los años formativos no se utilizan en su puesto de $D$ 
trabajo. Los problemas de desajuste educativo y de desajuste en competencias resultan así de especial interés desde la perspectiva de las políticas públicas, con el objetivo de tratar de mejorar el ajuste entre la oferta y la demanda del capital humano en el mercado de trabajo, lo que permitiría tanto a los individuos como a la economía en su conjunto alcanzar un mayor rendimiento de la inversión realizada a través de las políticas públicas en educación.

El capital humano de los individuos y sus efectos económicos son cuestiones que han suscitado el interés de muchos investigadores a lo largo del tiempo. Tradicionalmente, el capital humano se ha aproximado a través de los años de escolaridad o del nivel educativo alcanzado por los individuos, encontrando que la educación tiene efectos positivos y significativos sobre determinados outcomes laborales como pueden ser los salarios y la satisfacción laboral. Sin embargo, en las últimas décadas, ha cobrado importancia el estudio de las competencias cognitivas efectivamente adquiridas por los individuos como parte de su capital humano, destacando la importancia de las competencias alcanzadas sobre el rendimiento salarial (McIntosh y Vignoles, 2001; Green y Riddell, 2003; Mateos-Romero et al., 2014).

También son muchos los trabajos que analizan qué sucede cuando el nivel educativo o el nivel de competencias de los individuos no se corresponden con los requerimientos del puesto de trabajo. En el caso del desajuste educativo, la literatura muestra que la sobreeducación tiene consecuencias negativas sobre los salarios de los trabajadores (Hartog y Oosterbeck, 1988; Kiker et al., 1997; Dolton y Vignoles, 2000). Por su parte, recientemente también se han estudiado los efectos del desajuste en competencias, encontrando que una infrautilización de las mismas puede conllevar a penalizaciones salariales (Allen y Van der Velden, 2001; DiPietro y Urwin, 2006). Estos resultados son, en principio, acordes con la literatura del desajuste educativo ya que la sobreeducación suele asociarse a una infrautilización de las competencias en el puesto de trabajo.

Sin embargo, diversos estudios que analizan el desajuste educativo y el desajuste en competencias conjuntamente tienden a destacar que estos desajustes reflejan distintos fenómenos (Allen y Van der Velden, 2001; Green y Mclntosh, 2007). La principal explicación para esta falta de correspondencia entre desajuste educativo y desajuste en competencias se basa en la hipótesis de heterogeneidad de competencias, que destaca cómo individuos con un mismo nivel educativo pueden alcanzar distintos niveles de competencias. De ser así no existiría una correspondencia unívoca entre niveles educativos y competencias adquiridas $\mathrm{y}$, consecuentemente, un desajuste educativo no se traduciría necesariamente en un desajuste en competencias.

En este contexto, el principal objetivo de este trabajo es revisar la literatura sobre los desajustes encontrados en el mercado laboral, prestando especial atención al nivel de competencias alcanzado por los individuos como parte de su capital humano. De esta forma, en el apartado siguiente se estudian los efectos económicos del capital humano, aproximado tanto por la educación recibida como por las competencias alcanzadas. Por su parte, el apartado 3 se centra en los desajustes observados en el mercado laboral, ya se trate de desajustes educativos o en competencias. En este apartado se revisan distintos marcos teóricos propuestos para analizar estos desajustes, las principales formas de medición y los principales resultados empíricos alcanzados con relación a sus efectos sobre los salarios. EI $\triangleright$ 
apartado 4 revisa aquellos estudios que consideran competencias heterogéneas entre los individuos que cuentan con un mismo nivel educativo y distinguen entre sobreeducación real y aparente, en función de los niveles de competencias adquiridas por los individuos. Por último, el trabajo se cierra con un breve apartado de conclusiones.

\section{Educación y competencias cognitivas}

Los efectos económicos de la educación han sido ampliamente estudiados por los economistas desde el desarrollo de la teoría del capital humano a mediados de los años sesenta. Estos efectos han sido investigados tanto en el ámbito macroeconómico como en el ámbito microeconómico. Desde una perspectiva macroeconómica se destaca el papel fundamental que desempeña el capital humano a la hora de explicar la productividad y el crecimiento económico (Barro, 1991; Mankiw et al. 1992). Por su parte, en el ámbito microeconómico se destaca la rentabilidad individual del capital humano en términos de acceso y condiciones de empleo y de mayores salarios (véase Card, 1999; y Harmon et al., 2003).

La mayor parte de estas investigaciones utilizan los años de escolaridad de los individuos o el nivel de estudios alcanzado como proxy del capital humano. Sin embargo, en ocasiones se señala que los años de escolaridad o los niveles de estudio no reflejan efectivamente las competencias cognitivas realmente adquiridas. En este sentido, en los últimos años han aparecido una serie de encuestas estandarizadas que permiten una mejor aproximación y medición de las competencias alcanzadas por los individuos.
En concreto, desde mediados de los años noventa del pasado siglo, la OCDE ha desarrollado tres programas para medir las competencias adquiridas por la población adulta. El primero de ellos fue el International Adult Literacy Survey (IALS). Los test IALS nacieron en 1994 para obtener la primera escala comparativa a nivel mundial de la medición de las competencias de alfabetización y literatura de los adultos de los países participantes. En la primera encuesta realizada participaron Canadá, Francia, Alemania, Irlanda, Países Bajos, Polonia, Suecia, Suiza y Estados Unidos. Dada la gran acogida de los resultados obtenidos, otros territorios (Australia, Bélgica, Gran Bretaña, Nueva Zelanda e Irlanda del Norte) se unieron a la segunda oleada de estos test en 1996. En la última oleada de 1998 llegaron a participar 22 países o regiones.

Por su parte, la encuesta Adult Literacy and Lifeskills (ALL) se desarrolló entre los años 2003 y 2006 con el objetivo de proporcionar a los países participantes información acerca de las competencias de su población adulta. Esta encuesta partía de una muestra representativa de cada país que incluía a personas entre 16 y 65 años para medir sus competencias en matemáticas y literatura. En la primera oleada de estos test únicamente participaron seis países: Bermuda, Canadá, Italia, Noruega, Suiza y Estados Unidos. En la segunda ronda se unieron además Australia, Hungría, Países Bajos y Nueva Zelanda.

Por último, el Programme for the International Assessment of Adult Competencies (PIAAC) es el tercero y más reciente de los test desarrollados por la OCDE que tratan de medir las competencias de los adultos. PIAAC nace con el objetivo de estudiar las competencias de los individuos, ofreciendo además información acerca de cómo los adultos utilizan las competencias en su hogar, en el lugar de $\triangleright$ 
trabajo y en la sociedad en general. PIAAC ha sido diseñado como un programa permanente de evaluación. El primer ciclo de este programa se ha planteado en dos rondas. La primera de ellas se llevó a cabo desde enero de 2008 hasta octubre de 2013 y participaron 24 países: Australia, Austria, Canadá, Chipre, República Checa, Dinamarca, Reino Unido, Estonia, Finlandia, Bélgica, Francia, Alemania, Irlanda, Italia, Japón, Corea, Países Bajos, Noruega, Polonia, Rusia, República Eslovaca, España, Suecia y Estados Unidos. La segunda ronda del primer ciclo se inició en 2012 y se ha completado en 2016 con la participación de nueve países adicionales (Chile, Grecia, Indonesia, Israel, Lituania, Nueva Zelanda, Singapur, Eslovenia y Turquía).

Con el desarrollo de estos programas, el estudio de los efectos económicos del capital humano ha avanzado recientemente incorporando estas nuevas medidas de competencias al análisis. De esta forma, a nivel macroeconómico, diversos trabajos sobre capital humano y crecimiento económico destacan el papel que juegan las competencias realmente adquiridas, y no solo los años de escolarización, en el crecimiento de la productividad (Hanushek y Kimko, 2000; Barro, 2001; Hanushek y Woessmann, 2008). Por su parte, en el ámbito microeconómico, la evidencia empírica confirma también los efectos positivos de las competencias alcanzadas por los trabajadores sobre la probabilidad de empleo y sobre los salarios (véanse, por ejemplo, Mclntosh y Vignoles, 2001; o Green y Riddell, 2003). En definitiva, la evidencia empírica tiende a confirmar que tanto la educación recibida (medida en años o en niveles educativos alcanzados) como las competencias adquiridas presentan efectos positivos y significativos sobre distintos outcomes económicos.

\section{Desajuste educativo y desajuste en competencias}

\subsection{Marcos teóricos}

Desde el lado de la oferta en el mercado de trabajo, una de las primeras teorías que nos permite considerar el grado de ajuste en el mercado laboral es la teoría del capital humano. La teoría del capital humano comienza a desarrollarse a partir de mediados de los años sesenta gracias a las pioneras contribuciones de Schultz (1961) y Becker (1964). Dicha teoría concibe los gastos en educación y formación como una inversión en capital humano que incrementará la productividad de los individuos. Por su parte, puesto que la productividad viene determinada por el capital humano acumulado por el individuo, la sobreeducación se ve como un fenómeno temporal que solamente tiene cabida en el corto plazo, es decir, mientras las empresas adaptan los procesos de producción a la fuerza de trabajo para aprovechar el capital humano de sus empleados, o mientras los trabajadores encuentran un trabajo que se adapte a su nivel de estudios.

De forma similar, la teoría de la movilidad (Sicherman y Galor, 1990) también entiende la sobreeducación como un fenómeno temporal. En este caso, se explica en el corto plazo porque los trabajadores aceptan puestos de trabajo para loS que están sobreeducados con el fin de adquirir experiencia y capital humano específico. En el largo plazo, cuando ya hayan obtenido dicha experiencia, se asume que encontrarán un puesto más adecuado a su nivel educativo.

La teoría más extendida para explicar la sobreeducación desde el lado de la demanda de trabajo es la teoría de la competencia laboral (Thurow, 1975). Esta teoría considera que los $\triangleright$ 
salarios vienen determinados por el puesto de trabajo, de forma que los empleadores fijan el salario de sus empleados en base a las características observables de los puestos de trabajo, sin tener en cuenta el rendimiento personal de cada individuo. Además, esta teoría contempla el mercado laboral como un mercado de formación, es decir, considera que la mayoría de las competencias laborales se adquieren en el puesto de trabajo y no mediante la educación formal. En esta situación, los individuos llevan a cabo inversiones en educación no con el objetivo de aumentar su productividad como sugiere la teoría del capital humano, sino para competir por los mejores puestos de trabajo. Con esta teoría, la sobreeducación se explica como una respuesta de los individuos para defender su posición relativa en el mercado laboral.

En la misma línea encontramos la teoría de la señalización (Spence, 1973), que sugiere que los individuos invierten en educación no para aumentar su productividad, sino para enviar señales sobre su capacidad. Según esta teoría los empleadores toman el nivel educativo de los individuos como referencia y se crean sus propias expectativas de productividad de los trabajadores en función del nivel educativo que hayan alcanzado. En este contexto, los individuos podrían realizar una sobreinversión en educación con el objetivo de enviar una mejor señal sobre sus capacidades a los empleadores.

Por su parte, la teoría de la asignación (Sattinger, 1993) tiene en cuenta tanto el lado de la oferta como el lado de la demanda del mercado de trabajo. De esta forma, los salarios se determinan en parte por las características de los puestos de trabajo y en parte por las características productivas de los individuos. Por tanto, las inversiones adicionales en capital humano y el rendimiento de esa inversión dependerán de la combinación entre puesto de trabajo y trabajador. Esta teoría entiende que la sobreeducación puede ser un problema persistente en el largo plazo, puesto que es consecuencia de los problemas en la distribución y asignación de los puestos de trabajo a cada individuo. Como resultado de dicho proceso de asignación, algunos trabajadores ocupan puestos para los que no presentan ventajas comparativas, lo que se traduce en una ganancia de salarios más baja.

Las diferencias salariales se explican considerando que el puesto de trabajo limita e impone un techo a la productividad del individuo. El grado en que los trabajadores pueden utilizar sus competencias va a depender, al menos en parte, de las características del puesto de trabajo. Por tanto, según esta teoría, si un individuo ocupa un puesto de trabajo cuyo nivel requerido de educación es inferior al que posee el individuo (sobreeducación), el techo a la productividad que impone el puesto de trabajo no le permitirá desarrollar al máximo todas sus competencias, lo que redundará en un menor salario que si ocupase un puesto acorde a su formación. Por el contrario, un trabajador cuyo puesto de trabajo requiere un mayor nivel educativo que el poseído por el individuo (infraeducación) tiene limitada la productividad por sus menores competencias, si bien los mayores requerimientos de su puesto de trabajo le permitirán obtener un mayor salario que el que obtendría en un puesto de trabajo cuyos requerimientos fueran menores.

\subsection{Formas de medición del desajuste}

A la hora de estudiar el desajuste educativo y en competencias se han venido utilizando, en la literatura empírica, distintas formas de $\triangleright$ 
medición que pueden clasificarse en tres grandes grupos: métodos objetivos, métodos subjetivos y métodos estadísticos.

El método objetivo se basa en el análisis de expertos externos que examinan diferentes aspectos de los distintos grupos ocupacionales con el fin de establecer una escala con las características de cada grupo, la formación y experiencia necesaria, el grado de dificultad de las tareas a realizar, el tipo y nivel de educación conveniente, etcétera. Una vez se tengan los requerimientos necesarios para cada grupo ocupacional se comparan con las características del trabajador para establecer si existe o no desajuste.

La principal ventaja de este método es que se trata de medidas objetivas y exógenas. Sin embargo, en ocasiones se han criticado estas medidas ya que no pueden considerar toda la heterogeneidad de las ocupaciones y además los requerimientos de las distintas ocupaciones pueden variar en un corto plazo de tiempo. Hartog (2000) destaca los inconvenientes que puede presentar este método en relación con la medición del desajuste educativo, aunque también sería aplicable al desajuste en competencias. En concreto, señala que este método necesita mucha información costosa y difícil de conseguir y puede presentar problemas metodológicos si no existe una escala de conversión para comparar la educación de los individuos con las exigencias establecidas para cada puesto de trabajo.

En el caso de los métodos subjetivos, es el trabajador el que ha de considerar, de forma directa o indirecta, si su puesto de trabajo se ajusta o no a su educación (o si su puesto de trabajo se adecúa o no a sus competencias). Cuando hablamos de medidas subjetivas directas nos referimos a aquellas en las que se le pregunta al individuo explícitamente si piensa que está sobreeducado, infraeducado o convenientemente educado en relación con el puesto de trabajo que desempeña. Del mismo modo, serían medidas subjetivas directas del desajuste en competencias aquellas en las que se pregunta directamente si su empleo se ajusta a sus competencias. En cambio, las medidas subjetivas indirectas radican en preguntar al trabajador acerca del nivel educativo que supone óptimo para desempeñar su trabajo con el fin de, posteriormente, comparar estas respuestas con el nivel de formación del individuo. Como medidas subjetivas indirectas del desajuste en competencias se utilizan diversas preguntas acerca de si serían necesarias o no mayores competencias para realizar su trabajo y se comparan, posteriormente, con las competencias que realmente poseen los individuos.

A este procedimiento se le suelen atribuir numerosas ventajas. Por ejemplo, salva uno de los inconvenientes que presentan los métodos objetivos y es que no se refiere a un grupo ocupacional, sino que tiene en cuenta puestos de trabajos específicos (Hartog, 2000). Además, se considera que es el trabajador el que mejor conoce las características del puesto. Sin embargo, este hecho también puede considerarse como un inconveniente ya que se trata de una percepción del individuo que puede incluir aspectos emocionales (Hartog y Oosterbeek, 1988; Mendes de Oliveira et al., 2000).

Por último, el método estadístico se basa en ajustes realizados, aproximados por la media $o$ la moda del nivel educativo observado en una ocupación determinada. Con este método se calcula, en primer lugar, la media/moda de años de educación formal de los trabajadores de una ocupación y a continuación se compara con los años de educación del individuo. Para determinar el desajuste educativo se comprueba si la educación de los individuos se encuentra $\triangleright$ 
dentro del intervalo formado por la media (o la moda) más/menos una desviación típica. Si se encuentra dentro de dicho intervalo no existe desajuste educativo, mientras que si la educación del individuo se sitúa por encima o por debajo de los límites del intervalo estaríamos ante sobreeducación o infraeducación, respectivamente. Entre las principales ventajas del método estadístico se puede destacar que es un sistema que necesita poca información y fácil de conseguir, si bien la utilización de la desviación típica como referencia (o la elección de algún otro intervalo) resulta una medida arbitraria.

Hartog (2000) realiza una comparativa de estos tres métodos concluyendo que el método objetivo es la mejor opción teórica, mientras que el procedimiento subjetivo puede ser el más adecuado en la práctica debido, sobre todo, a la información disponible. Green et al. (1999) y Groot y van der Brink (2000) establecen que no existen diferencias significativas en los resultados como consecuencia del método de medición que se utiliza. En conclusión, la elección de un método u otro tendrá que tener en cuenta aspectos tales como la información disponible, el nivel de desagregación de los datos o la actualización de los mismos.

\subsection{Desajuste educativo y desajuste en competencias: evidencia empírica}

El origen del concepto de sobreeducación generalmente se asocia a la investigación realizada por Freeman (1976), quien encontró un descenso en los rendimientos de la educación entre los universitarios estadounidenses. Según este autor, un incremento de oferta de mano de obra cualificada, unido a una moderación en su demanda, hizo que aumentara el número de trabajadores sobreeducados.
A partir de este estudio han aparecido numerosos trabajos que analizan el fenómeno de la sobreeducación y sus efectos sobre los salarios. En el estudio de Hartog y Oosterbeek (1988) se utilizan datos de 1982 relativos a 1.182 individuos con el objetivo de estimar tanto el desajuste educativo como el rendimiento de la educación en los Países Bajos. Para estimar los años de sobreeducación y de infraeducación hacen uso del método objetivo a partir de la clasificación del Dutch Department of Social Affairs y del ARBI code. En su estudio estiman tres ecuaciones: una primera ecuación en la que se relacionan los salarios con los años de escolaridad, otra en la que tienen en cuenta los años requeridos para el puesto de trabajo y una última especificación que incluye tanto los años de sobreeducación como los de infraeducación. Sus resultados muestran que la rentabilidad de la educación para los individuos sobreeducados es inferior a la rentabilidad de los trabajadores adecuadamente educados mientras que los trabajadores infraeducados obtienen una penalización del 2,5 por 100.

Verdugo y Verdugo (1989) estudian el efecto de la sobreeducación en los salarios. Utilizan una muestra de 14.596 estadounidenses de entre 25 y 64 años para estimar unas ecuaciones en las que se tiene en cuenta los años de escolarización, la experiencia y una variable dummy que mide la sobreeducación de los individuos. Además, también incluyen una serie de variables que hacen referencia a aspectos personales y características de los puestos de trabajo. Para medir la sobreeducación, dichos autores utilizan el método estadístico del intervalo sobre la media. Sus resultados muestran que los trabajadores sobreeducados ganan aproximadamente un 13 por 100 menos que aquellos con el mismo nivel de estudio pero que poseen un empleo acorde a su $\triangleright$ 
cualificación; mientras que los infraeducados ganan aproximadamente un 10 por 100 más que los individuos que presentan consonancia entre trabajo y escolaridad.

Dolton y Vignoles (2000) usan la National Survey of Graduates and Diplomates de 1980 de Reino Unido para poner a prueba la teoría del capital mediante el contraste de dos hipótesis: (1) si la educación requerida para el puesto y la sobreeducación producen los mismos rendimientos y (2) si los sobreeducados con un mayor nivel de educación ganan más que los sobreeducados con un menor nivel de educación. Estos autores, para el método subjetivo, preguntan a los individuos cuál sería el nivel mínimo de educación necesario para desempeñar su trabajo y lo comparan con el que poseen. Una vez obtenidas estas variables, estiman ecuaciones salariales que rechazan las dos hipótesis anteriores, de forma que comprueban que la sobreeducación es un fenómeno que se mantiene en el largo plazo y que reduce los salarios de los individuos.

En España, uno de los estudios más recientes es el de Murillo et al. (2012) en el que además de analizar el desajuste educativo y el rendimiento de la educación, se estudia la evolución de dichos rendimientos. Para ello utilizan la Encuesta de Estructura Salarial en las oleadas de 1996, 2002 y 2006. Su análisis del desajuste educativo mediante la estimación de ecuaciones mincerianas concluye que el rendimiento de la educación en España ha decrecido a lo largo del período analizado. Por otro lado, para estimar el rendimiento de la educación, en función de su grado de ajuste con el empleo desempeñado, utilizan las especificaciones ORU (Over-Required-Undereducated). La medición de los años de sobreeducación se realiza mediante el método del intervalo sobre la moda. Sus resultados muestran que el rendimiento económico de un año adicional de sobreeducación es positivo pero inferior a la rentabilidad de un año adicional de educación requerida. En cambio, el rendimiento económico de un año de infraeducación resulta negativo.

En definitiva, las investigaciones que se han llevado a cabo en la mayoría de países desarrollados encuentran que la sobreeducación afecta a una parte importante de la población educada con consecuencias negativas sobre los salarios. Estos efectos negativos se manifiestan en penalizaciones salariales para los individuos sobreeducados en comparación con otros individuos que, con su mismo nivel educativo, ocupan un puesto de trabajo acorde a su formación, o en primas salariales positivas para los años de sobreeducación cuando la comparación se realiza con aquellos trabajadores que ocupan un puesto de trabajo similar, si bien el rendimiento para estos años de sobreeducación es inferior al correspondiente a los años de educación requerida para desempeñar el puesto de trabajo ${ }^{1}$.

Una línea de investigación más reciente analiza la relación existente entre el desajuste educativo y las competencias de los individuos. Un trabajo pionero en comprobar si el desajuste educativo implica desajuste en competencias es el realizado por Allen y van der Velden (2001). Utilizando una muestra de individuos holandeses con estudios superiores, el objetivo de su trabajo es poner a prueba la teoría de la asignación, que supone que los individuos sobreeducados están infrautilizando sus competencias y de ahí su penalización salarial. Tanto para la medición del desajuste educativo como para el desajuste en competencias $\square$

1 Una revisión detallada sobre los efectos de la sobreeducación puede encontrarse en Groot y Maasen van der Brink (2000), Sloane (2003), McGuinnes (2006) o Leuven y Oosterbeek (2011). 
emplean el método subjetivo indirecto. Las competencias que consideran son unas competencias generales en relación con el puesto de trabajo procedentes de dos preguntas: una de ellas se centra en medir si el empleo del individuo le permite utilizar las competencias que posee y la otra trata de evaluar si existe un déficit de competencias. Aunque encuentran que un pequeño porcentaje de graduados experimenta una alta infrautilización de competencias, al analizar las respuestas de los individuos sobreeducados observan que una gran proporción de estos declara poca o ninguna infrautilización de sus competencias. Al introducir tanto las variables de desajuste educativo como las variables de desajuste en competencias en una regresión salarial, se comprueba que el desajuste educativo tiene un mayor impacto sobre los salarios que el desajuste en competencias.

Un trabajo similar es el realizado por DiPietro y Urwin (2006) con individuos italianos para probar en qué medida la sobreeducación refleja infrautilización de competencias. En este caso, también trabajan únicamente con universitarios y, mediante el método subjetivo indirecto, crean una variable mediante la cual establecen qué individuos utilizan (o no) las competencias adquiridas durante sus estudios en la universidad en su empleo actual. Hallan una fuerte correlación entre desajuste educativo y desajuste en competencias que se ve reflejada en el hecho de que cerca del 73 por 100 de graduados adecuadamente educados considera que está utilizando muchas de sus competencias en su trabajo actual. Sin embargo, al comprobar el uso de las competencias entre los sobreeducados, encuentran que solo el 20 por 100 declara sufrir infrautilización de las mismas. Cuando se introducen conjuntamente variables de desajuste educativo y en competencias en una regresión salarial, la penalización sufrida por los trabajadores sobreeducados no se reduce, lo que sugiere que el desajuste educativo no actúa a través del desajuste en competencias.

En la misma línea se encuadra la investigación realizada por Green y McIntosh (2007) con trabajadores británicos. Aplicando el método subjetivo indirecto, utilizan varias preguntas para medir el nivel de competencias generales que los individuos pueden utilizar en su puesto de trabajo. Al cruzar las variables de desajuste educativo y de desajuste en competencias, se halla una correlación positiva entre ellas, aunque se trata de una correlación débil. En la distribución de individuos sobreeducados encuentran que el 47 por 100 de los mismos poseen competencias que no están utilizando en su puesto de trabajo actual. Al igual que los autores anteriores, Green y McIntosh no encuentran disminución de la penalización salarial de los sobreeducados al considerar simultáneamente desajuste educativo y desajuste en competencias en una misma regresión salarial.

De esta forma, los estudios que analizan conjuntamente el desajuste educativo y el desajuste en competencias tienden a contradecir la teoría de la asignación en el sentido de que la penalización salarial de los trabajadores sobreeducados no parece deberse a una infrautilización de sus competencias. Consecuentemente, estos trabajos concluyen que el desajuste educativo y el desajuste en competencias son dos fenómenos distintos.

\section{Heterogeneidad de competencias: sobreeducación real y aparente}

Las formas tradicionales de medición del capital humano de los individuos y del $\triangleright$ 
desajuste educativo se basan en el supuesto de homogeneidad de competencias entre los trabajadores con un mismo nivel educativo. Este supuesto implícito de homogeneidad de competencias considera que los individuos que alcanzan un mismo nivel educativo cuentan con unas competencias similares $y$, por tanto, son sustitutos perfectos. La creciente insatisfacción con este supuesto ha dado lugar, en los últimos años, a una nueva literatura que considera la posibilidad de que individuos con un nivel educativo similar alcancen distintos niveles de competencias. Este hecho implica que el desajuste que muestran algunos trabajadores puede no ser real, sino que puede tratarse de un desajuste aparente al no existir correspondencia entre su nivel educativo y el nivel de competencias adquiridas. De esta forma, los individuos con un mayor nivel educativo que el requerido para su puesto de trabajo, pero que posean unas competencias acordes a dicho puesto, presentarán solamente sobreeducación formal o aparente. En cambio, si tienen unas competencias superiores serán trabajadores con sobreeducación genuina o real.

Dentro de esta nueva literatura sobre competencias heterogéneas, algunos autores han propuesto definiciones alternativas para la medición del desajuste educativo con la idea de distinguir entre sobreeducación real y aparente de acuerdo a las competencias con que cuentan efectivamente los individuos. En este sentido, encontramos la propuesta de Chevalier (2003), según la cual se distingue entre individuos real y aparentemente sobreeducados, en función de la satisfacción entre las necesidades educativas del puesto de trabajo y del nivel de estudios. De este modo, los trabajadores que muestren insatisfacción serán los que presenten sobreeducación real y los que se declaren satisfechos serán aparentemente sobreeducados. Por su parte, Green y Zhu (2010) miden la adecuación de las competencias generales de los individuos con el puesto de trabajo que ocupan y consideran que aquellos trabajadores que presentan un exceso de competencias estarán realmente sobreeducados mientras que aquellos con competencias adecuadas serán aparentemente sobreeducados. Por último, Mateos-Romero y Salinas-Jiménez (2017) utilizan directamente las competencias adquiridas por los individuos, considerando que un trabajador sobreeducado presenta sobreeducación real si posee unas competencias que corresponden con su nivel de estudios mientras que si tiene unas competencias inferiores será aparentemente sobreeducado.

Chevalier (2003) fue pionero en ofrecer una definición alternativa que contempla la heterogeneidad de trabajadores dentro de un mismo nivel educativo. En su estudio utiliza una muestra de individuos universitarios de Reino Unido y los clasifica como adecuadamente educados si están empleados en una ocupación cualificada y como sobreeducados en caso contrario. Posteriormente, como proxy de las competencias, recurre a una variable de satisfacción entre el nivel educativo y el puesto de trabajo. De esta forma, aquellos trabajadores sobreeducados que se declaren satisfechos con esa relación estarán aparentemente sobreeducados mientras que, en caso contrario, la sobreeducación será real. Con esta distinción entre real y aparente estima una regresión salarial cuyos resultados apuntan a que los trabajadores realmente sobreeducados sufren una penalización mayor que los aparentemente sobreeducados. Además, su encuesta contiene un componente longitudinal que le permite crear una variable que incorpora como proxy de las competencias no observadas. Para ello, estima los salarios $\square$ 
del primer empleo y supone que la diferencia entre el salario esperado y el observado es una aproximación a dichas características no observables. Al introducir esta variable explicativa en la regresión anterior, la estimación de las penalizaciones apenas varía.

Esta forma de medición del desajuste ha sido utilizada por otros autores para mostrar la existencia de competencias heterogéneas entre los trabajadores con el mismo nivel educativo. Chevalier y Lindley (2009) realizan un estudio en Reino Unido en el que, además de distinguir entre trabajadores real y aparentemente sobreeducados, incluyen como variables explicativas en la regresión salarial el hecho de que un individuo tenga exceso o déficit de competencias. También examinan las competencias no observadas gracias al componente longitudinal de la base de datos utilizada. Al estimar los efectos salariales del desajuste con estas variables, y controlando tanto las competencias observadas como las no observadas, se encuentran penalizaciones salariales significativas para ambos grupos de sobreeducados, siendo mayor la sufrida por los individuos realmente sobreeducados.

Una propuesta alternativa para considerar la posibilidad de competencias heterogéneas es la de Green y Zhu (2010), quienes utilizan el desajuste en competencias percibido por los individuos para distinguir entre sobreeducación real y aparente. Estos autores trabajan con una muestra de individuos británicos de entre 25 y 60 años procedente de la encuesta 2006 Skills Survey y de tres de sus oleadas anteriores. Utilizan el método subjetivo indirecto para clasificar a los individuos en sobreeducados, adecuadamente educados, e infraeducados. En dicha encuesta disponen de dos preguntas que permiten medir la adecuación de las competencias de los trabajadores con su puesto de trabajo. La primera de ellas hace referencia a qué parte de la experiencia pasada pueden utilizar los individuos en su empleo actual. Por su parte, la segunda pregunta se centra en medir si el puesto de trabajo permite al trabajador utilizar todas sus competencias y habilidades. En función de las respuestas a estas preguntas clasifican a los individuos como realmente sobreeducados si infrautilizan sus competencias y como formalmente sobreeducados en caso contrario. Los resultados de su distribución muestran que existe un mayor porcentaje de individuos cuya sobreeducación es solamente formal (28 por 100 frente a 10 por 100 aproximadamente). Tras incluir las variables de sobreeducación en una regresión salarial encuentran que la penalización salarial para los trabajadores que muestran sobreeducación real es mayor que la de los individuos con sobreeducación aparente.

Mavromaras et al. (2013) parten también de esta idea de infrautilización de competencias para considerar la hipótesis de heterogeneidad. Estos autores trabajan con datos de panel procedentes de siete oleadas de la encuesta Household Income and Labour Dynamics in Australia (HILDA). Su muestra está compuesta por universitarios australianos de entre 16 y 64 años que se encuentran asalariados a tiempo completo. Los datos disponibles en esta encuesta permiten medir tres aspectos del desajuste educativo: sobreeducación, exceso de competencias y ambos fenómenos simultáneamente. Para la medición de la sobreeducación aplican el método estadístico, tomando el valor modal de los años de escolaridad como referencia. Por su parte, el exceso de competencias lo miden preguntando directamente a los trabajadores si utilizan sus competencias y habilidades en su trabajo actual. Para comprobar el efecto de estos fenómenos sobre $\square$ 
los salarios, se estiman regresiones salariales teniendo en cuenta las variables definidas anteriormente. Los datos de panel les permiten, además de estimar las regresiones por mínimos cuadrados ordinarios (MCO), aplicar el procedimiento de efectos fijos para controlar la heterogeneidad no observada. Con MCO se estima una penalización salarial para los individuos sobreeducados, en torno al 21 por 100 , mientras que para aquellos trabajadores que también sufren exceso de competencias la penalización estimada alcanza el 31 por 100. Al aplicar los estimadores de efectos fijos siguen valorándose penalizaciones diferentes, aunque estas se ven reducidas.

Distinguiendo también sobreeducación real y aparente en función del grado de utilización de las competencias generales de los trabajadores, se encuentra la investigación realizada por Pecoraro (2014). Su muestra de 1.370 individuos procede de la Swiss Graduate Survey, una encuesta de carácter longitudinal realizada por universitarios al año y a los cinco años de finalizar sus estudios. Para contrastar la hipótesis de heterogeneidad de competencias, distingue inicialmente entre trabajadores adecuadamente educados y sobreeducados mediante el método subjetivo. A continuación, utiliza dos preguntas de su encuesta para diferenciar entre individuos real y aparentemente sobreeducados (y lo mismo para los adecuadamente educados). Estas dos preguntas miden el nivel de correspondencia que existe entre las competencias generales del individuo y su puesto de trabajo. De este modo, los trabajadores realmente sobreeducados serán aquellos que declaren unas competencias inadecuadas $y$ aparentemente sobreeducados en el caso contrario. Estas variables se incluyen en una ecuación minceriana y, al disponer de una base de datos longitudinal, se aplica además el método de efectos fijos y el de efectos aleatorios. Con mínimos cuadrados ordinarios agrupados, las estimaciones arrojan una penalización del 15,8 por 100 para los individuos realmente sobreeducados frente al 8,3 por 100 de los aparentemente. Con los métodos de efectos fijos y de efectos aleatorios las penalizaciones se reducen pero no desaparecen. Las distintas penalizaciones salariales encontradas están acordes a la hipótesis de heterogeneidad.

Por último, Mateos-Romero y Salinas-Jiménez (2017) relajan el supuesto de homogeneidad de competencias al analizar la sobreeducación con una muestra de trabajadores universitarios en España utilizando la base de datos PIAAC. Estas autoras proponen una nueva forma de distinguir entre sobreeducación real y aparente basándose directamente en las competencias adquiridas por los trabajadores y tomando como referencia bien el nivel educativo del individuo, bien su categoría ocupacional. En el caso del nivel educativo, se considera que un trabajador sobreeducado presenta sobreeducación real si posee unas competencias apropiadas a su nivel de estudios. Por el contrario, si tiene competencias inferiores será aparentemente sobreeducado. Para determinar qué nivel de competencias es el adecuado para su nivel educativo, se toma como referencia el valor medio del nivel de competencia lingüística o matemática de los individuos que, con el mismo nivel educativo, poseen un trabajo acorde a su formación. La segunda alternativa es considerar como referencia el nivel de competencias de los trabajadores de la misma categoría ocupacional. Así, los trabajadores sobreeducados que muestren un nivel de competencias adecuado para esa categoría ocupacional serán aparentemente sobreeducados mientras que si sus competencias son mayores se $\triangleright$ 
tratará de sobreeducación real. En este caso, para establecer el nivel de competencias que se considera apropiado para el puesto de trabajo se considera el valor medio del nivel de competencia lingüística o matemática de los trabajadores que poseen el nivel educativo necesario para el puesto de trabajo que ocupan. Para contrastar la existencia de dicha heterogeneidad entre los trabajadores con nivel educativo superior en España se introducen las variables de sobreeducación real y aparente en varias regresiones salariales. Cuando se toma como referencia el nivel educativo de los individuos se estiman diferentes penalizaciones salariales para la sobreeducación real y aparente. Del mismo modo, al establecer la comparación con los trabajadores de la misma categoría ocupacional, se estiman primas salariales positivas y significativas para la sobreeducación real y primas no significativas para la sobreeducación aparente. Ambos resultados sugieren la existencia de heterogeneidad de competencias entre los trabajadores con un nivel educativo superior.

\section{Conclusiones}

El estudio de los efectos económicos de la educación y el desajuste educativo tiene ya una larga tradición en la literatura económica. En la literatura empírica ha sido habitual aproximar el capital humano mediante variables referidas a la educación, aproximándolo por los años de educación cursados o por el nivel educativo alcanzado por los individuos. Sin embargo, en las dos últimas décadas han aparecido nuevas medidas que tratan de reflejar cuáles son las competencias cognitivas realmente adquiridas por los individuos, lo que ha dado un nuevo impulso a esta literatura.
El objetivo de este trabajo ha sido revisar esta literatura prestando especial atención a los desajustes observados en el mercado laboral y teniendo en consideración el nivel de competencias alcanzado por los individuos como parte de su capital humano. La revisión de los trabajos que analizan los efectos del capital humano sobre los salarios pone de manifiesto la relevancia tanto de la educación recibida como de las competencias efectivamente adquiridas en la determinación del rendimiento salarial. Por su parte, los estudios que analizan los efectos del desajuste educativo y del desajuste en competencias muestran que ambos presentan efectos negativos sobre los salarios. No obstante, los trabajos que analizan ambos fenómenos conjuntamente tienden a destacar cómo estos dos tipos de desajuste reflejan fenómenos distintos, sin que haya una correspondencia entre uno y otro en el mercado laboral.

Estos resultados sugieren que no hay una correspondencia unívoca entre educación recibida y competencias adquiridas. Esto es, que individuos con un mismo nivel educativo mostrarían niveles de competencias muy diferentes. Esto ha llevado en los últimos años a considerar la hipótesis de competencias heterogéneas entre individuos con niveles similares de educación, distinguiendo entre sobreeducación real y aparente en función del nivel de competencias efectivamente adquiridas por los trabajadores. Los resultados de esta nueva rama de la literatura sugieren que las penalizaciones del desajuste educativo difieren en función del nivel de competencias alcanzado por los individuos, apoyando así la hipótesis de heterogeneidad de competencias y poniendo de manifiesto la necesidad de considerar las diferencias en las competencias alcanzadas a la hora de estudiar los efectos del desajuste educativo. 


\section{Bibliografía}

[1] ALLEN, J. y VAN DER VELDEN, R. (2001). «Educational mismatches versus skill mismatches: effects on wages, job satisfaction, and on-the-job search». Oxford Economic Papers, vol. 53, n.․ 3, pp. 434-452.

[2] BARRO, R.J. (1991). «Economic Growth in a Cross Section of Countries». Quarterly Journal of Economics, vol. 106, pp. 407-443.

[3] BARRO, R.J. (2001). «Human Capital and Growth». American Economic Review, vol. 91, pp. $12-17$.

[4] BECKER, G. (1964). Human Capital. Nueva York: Columbia University Press.

[5] CARD, D. (1999). «The Causal Effects of Education on Earnings». En Handbook of Labor Economics (eds. Ashenfelter, A. y Card, D.), pp. 1801-1863. Amsterdam, North Holland.

[6] CHEVALIER, A. (2003). «Measuring OverEducation». Economica, vol. 70, n.을 279, pp. 509-531.

[7] CHEVALIER, A. y LINDLEY, J. (2009). "Overeducation and the skills of UK graduates». Journal of the Royal Statistical Society: Series A (Statistics in Society), vol. 172, n. .2 , pp. 307-337.

[8] DI PIETRO, G. y URWIN, P. (2006). «Education and skills mismatch in the Italian graduate labour market». Applied Economics, vol. 38, n. ${ }^{\circ}$ 1, pp. 79-93.

[9] DOLTON, P. y VIGNOLES, A. (2000). «The incidence and the effects of overeducation in the UK graduate labour market». Economics of Education Review, vol. 19, pp. 179-198.

[10] FREEMAN, R. (1976). The Overeducated American. New York: American Press.

[11] GREEN, F. y MCINTOSH, S. (2007). «Is there a genuine under-utilization of skills amongst the over-qualified? ». Applied Economics, vol. 39, n. 4 , pp. 427-439.

[12] GREEN, D. y RIDDELL, W.C. (2003). «Literacy and earnings: An Investigation of the Interaction of Cognitive and Unobserved Skills in Earnings Generation». Labour Economics, vol. 10, n.․ 2, pp. 165-184.

[13] GREEN, F., yZHU,Y.(2010). «Overqualification, job dissatisfaction, and increasing dispersion in the returns to graduate education». Oxford Economic Papers, vol. 62, n.․ 4, pp. 740-763.
[14] GREEN, F.; MCINTOSH, S. y VIGNOLES, A. (1999). "Overeducation and skills: clarifying the concepts". Centre for Economic Performance, Discussion Paper n.․ 435. London School of Economics.

[15] GROOT, W. y VAN DEN BRINK, H. (2000). "Overeducation in the Labor Market: A Metaanalysis». Economics of Education Review, vol. 19, pp. 145-158.

[16] HANUSHEK, E. y KIMKO, D. (2000). «Schooling, Labor Force Quality, and Economic Growth». American Economic Review, vol. 90, pp. 1184-1208.

[17] HANUSHEK, E. y WOESSMANN, L. (2008). «The Role of Cognitive Skills in Economic Development». Journal of Economic Literature, vol. 46, pp. 607-668.

[18] HARMON, C.; OOSTERBEEK, H. y WALKER, I. (2003). «The returns to education: Microeconomics». Journal of Economic Surveys, vol. 17, n. -2 , pp. 115-156.

[19] HARTOG, J. (2000). «Over-education and earnings: where are we, where should we go?». Economics of Education Review, vol. 19, n.. 2, pp. 131-147.

[20] HARTOG, J. y OOSTERBEEK, H. (1988). «Education, allocation and earnings in the Netherlands: Overschooling?». Economics of Education Review, vol. 7, n.ㅇ 2, pp. 185-194.

[21] KIKER, B.F.; SANTOS, M.C. y DE OLIVEIRA, M.M. (1997). "Overeducation and undereducation: Evidence for Portugal». Economics of Education Review, vol. 16, n.ㅇ 2, pp. 111-125.

[22] LEUVEN, E. y OOSTERBEEK, H. (2011). "Overeducation and mismatch in the labor market». Handbook of the Economics of Education, vol. 4, pp. 283-326.

[23] MANKIW, N.G.; ROMER, D. y WEIL, D.N. (1992). «A Contribution to the Empirics of Economic Growth». Quarterly Journal of Economics, vol. 107, pp. 407-437.

[24] MATEOS-ROMERO, L. y SALINASJIMÉNEZ (2017). "Skills Heterogeneity Among Graduate Workers: Real and Apparent Overeducation in the Spanish Labor Market». Social Indicators Research (DOI: 10.1007/s11205-016-1338-x).

[25] MATEOS ROMERO, L.; MURILLO HUERTAS, I.P. y SALINAS JIMÉNEZ, M.M. (2014). «Desajuste educativo y competencias cognitivas: $\triangleright$ 
efectos sobre los salarios». Hacienda Pública Española/Review of Publics Economics, vol. 210, n.. 3, pp. 85-108.

[26] MAVROMARAS, K.; MCGUINNESS, S.; O'LEARY, N.; SLOANE, P. y WEI, Z. (2013). «Job mismatches and labour market outcomes: Panel evidence on university graduates». Economic Record, vol. 89, n.․ 286, pp. 382-395.

[27] MCINTOSH, S. y VIGNOLES, A. (2001). «Measuring and assessing the impact of basic skills on labour market outcomes". Oxford Economic Papers, vol. 53, n.ㅇ 3, pp. 453-481.

[28] MCGUINNESS, S. (2006). "Overeducation in the labour market». Journal of Economic Surveys, vol. 20, n.ำ, pp. 387-418.

[29] MENDES DE OLIVEIRA, M.; SANTOS, M.C. y KIKER, B.F. (2000). «The role of human capital and technological change in overeducation». Economics of Education Review, vol. 19, n.으 2, pp. 199-206.

[30] MURILLO, I.P.; RAHONA, M. y SALINAS, M.M. (2012). «Effects of educational mismatch on private returns to education: An analysis of the Spanish case (1995-2006)». Journal of Policy Modeling, vol. 34, n. .5 , pp. 646-659.
[31] PECORARO, M. (2014). «Is there still a wage penalty for being overeducated but well-matched in skills? A panel data analysis of a Swiss graduate cohort». Labour, vol. 28, n.ㅇ 3, pp. 309-337.

[32] SATTINGER, M. (1993). «Assignment models of the distribution of earnings». Journal of economic literature. vol. 31, n. 2, pp. 831-880.

[33] SCHULTZ, T.W. (1961). «Investment in Human Capital». American Economic Review, vol. 51, pp. 1-17.

[34] SICHERMAN, N. y GALOR, O. (1990). «A theory of career mobility". Journal of Political Economy, vol. 98, pp. 169-192.

[35] SLOANE, P. J. (2003). «Much ado about nothing? What does the overeducation literature really tell us», en Buchel, de Grip y Mertens (Eds). Overeducation in Europe, pp. 11-49.

[36] SPENCE, M. (1973). «Job market signaling». The quarterly journal of Economics, vol. 87, n.ㅇ 3, pp. 355-374.

[37] THUROW, L.C. (1975). Generating inequality: Mechanisms of distribution in the US economy. New York: Basic books.

[38] VERDUGO, R.R. y VERDUGO, N.T. (1989). «The impact of surplus schooling on earnings: Some additional findings". Journal of Human Resources, vol. 24, n.ㅇ 4, pp. 629-643. 

ISSN 0214-8307

\section{SUSCRIPCIÓN ANUAL}

\begin{tabular}{|c|c|c|c|}
\hline \multicolumn{2}{|c|}{ BOLETÍN ECONÓMICO DE INFORMACIÓN COMERCIAL ESPAÑOLA (12 NÚMEROS) } \\
\hline & $\begin{array}{c}\text { ESPAÑA } \\
\mathbf{1} \text { año }\end{array}$ & $\begin{array}{c}\text { UNIÓN EUROPEA } \\
\mathbf{1} \text { año }\end{array}$ & $\begin{array}{c}\text { RESTO DEL } \\
\text { MUNDO } \\
\mathbf{1} \text { año }\end{array}$ \\
\hline SUSCRIPCIÓN & $65,00 €$ & $85,00 €$ & $85,00 €$ \\
\hline $\begin{array}{c}\text { Gastos de envío } \\
\text { España }\end{array}$ & $5,76 €$ & $24,36 €$ & $30,00 €$ \\
\hline $\begin{array}{c}\text { Más 4\% de IVA. } \\
\text { Excepto Canarias, Ceuta y Melilla }\end{array}$ & $2,83 €$ & & $115,00 €$ \\
\hline TOTAL & $\mathbf{7 3 , 5 9} €$ & $\mathbf{1 0 9 , 3 6} €$ & \\
\hline & & & \\
\hline
\end{tabular}

\section{EJEMPLARES SUELTOS}

\begin{tabular}{|c|c|c|c|}
\hline BOLETÍN ECONÓMICO & DE INFORMACIÓN & COMERCIAL ESPAÑOL & \\
\hline & $\begin{array}{l}\text { ESPAÑA } \\
1 \text { ejemplar }\end{array}$ & $\begin{array}{l}\text { UNIÓN EUROPEA } \\
1 \text { ejemplar }\end{array}$ & $\begin{array}{c}\text { RESTO DEL } \\
\text { MUNDO } \\
1 \text { ejemplar }\end{array}$ \\
\hline NÚMERO SUELTO & $7,00 €$ & $9,00 €$ & $9,00 €$ \\
\hline $\begin{array}{l}\text { Gastos de envío } \\
\text { España }\end{array}$ & $0,48 €$ & $2,03 €$ & $2,50 €$ \\
\hline $\begin{array}{l}\text { Más } 4 \% \text { de IVA. } \\
\text { Excepto Canarias, Ceuta y Melilla }\end{array}$ & $0,30 €$ & & \\
\hline TOTAL & $7,78 €$ & $11,03 €$ & $11,50 €$ \\
\hline \multirow[t]{2}{*}{ BOLETÍN ECONÓMICO } & DE INFORMACIÓN & COMERCIAL ESPAÑOL & \\
\hline & $\begin{array}{l}\text { ESPAÑA } \\
1 \text { ejemplar }\end{array}$ & $\begin{array}{l}\text { UNIÓN EUROPEA } \\
1 \text { ejemplar }\end{array}$ & $\begin{array}{c}\text { RESTO DEL } \\
\text { MUNDO } \\
1 \text { ejemplar }\end{array}$ \\
\hline $\begin{array}{l}\text { NÚMERO SUELTO } \\
\text { EXTRAORDINARIO }\end{array}$ & $12,00 €$ & $15,00 €$ & $15,00 €$ \\
\hline Gastos de envío España & $0,48 €$ & $2,03 €$ & $2,50 €$ \\
\hline $\begin{array}{c}\text { Más } 4 \% \text { de IVA. } \\
\text { Excepto Canarias, Ceuta y Melilla }\end{array}$ & $0,50 €$ & & \\
\hline TOTAL & $12,98 €$ & $17,03 €$ & $17,50 €$ \\
\hline
\end{tabular}

\section{DATOS}

Nombre y apellidos

Empresa

Domicilio

D.P.

N.I.F.

Teléf.

Email

\section{DATOS DEL EDITOR:}

NIF:S2800568D

Transferencia a la cuenta de ingresos por venta de publicaciones del Ministerio de Economía y Competitividad.

IBERCAJA. Calle Alcalá 29. 28014 MADRID (ESPAÑA) CÓDIGO CUENTA CLIENTE: 2085-9252-07-0330598330 CÓDIGO BIC DE IBERCAJA: CAZRES2Z

IBAN: ES47 2085-9252-07-0330598330

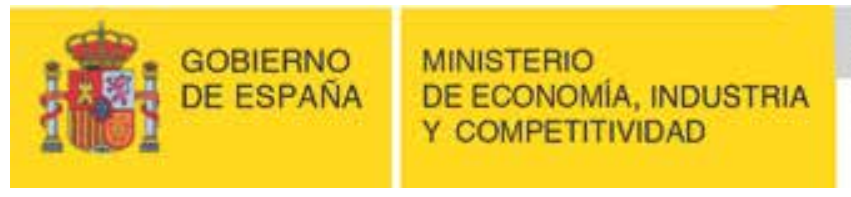

SUBSECAETARIA

SECRETARIA GENERAL TÉCNICA

SUBDIRECCION GENERAL DE

ESTUDIOS, INFORMACION Y PUBLICACIONES

Suscripciones y ventas por correspondencia:

Paseo de la Castellana, $1628^{\text {a }}$ Planta 28046 Madrid. Teléfono 916037993 / 97

Suscripciones a través de la página web del Ministerio de Economía, Industria y Competitividad distribucionpublicaciones@mineco.es 